\title{
The Nonlinear Optics of Plasmas
}

\author{
C. Joshi \\ University of California, Los Angeles, Los Angeles, CA 90024, U.S.A.
}

Received May 22, 1989

\begin{abstract}
It is well known that plasmas exhibit many of the characteristics of a non-linear optical medium when intense electromagnetic waves are incident upon it. These include harmonic generation, simulated Brillouin and Raman scattering, degenerate and resonant four wave mixing, self-focusing and parametric amplification. In addition, plasmas demonstrate certain unique nonlinear phenomena such as nonlinear mode conversion, two plasmon decay and relativistic self-focusing. The nonlinear optics of plasmas is therefore at once both challenging and rich for an experimentalist. It is challenging because many of the nonlinear optical phenomena occur simultaneously in a plasma. Also, when the plasma modes, characteristic of many of these instabilities, become large, kinetic effects and wave-wave interactions become extremely important. On the other hand, the plasma medium can be directly probed using coherent Thomson scattering technique revealing the richness of the physics. Experiments carried out by the author and his collaborators over the past decade are summarized and future directions for this field outlined in this paper.
\end{abstract}

\section{Introduction}

The pursuit of laser fusion has led to the development of extremely high peak power lasers during the last two decades. When such lasers are focused, extremely high intensities are generated. High intensities necessarily imply high electric fields, therefore any atoms within the focal volume are rapidly ionized and a plasma is formed. One parameter that characterizes the strength of the nonlinear interaction between the incident laser and the plasma is the oscillating velocity, $v_{0}$, of the electron in the electromagnetic field. This parameter can be expressed in terms of laser intensity and wavelength as,

$\frac{v_{0}}{c}=8.55 \times 10^{-10} \hat{\lambda}_{\mu} I\left(\mathrm{~W} / \mathrm{cm}^{2}\right)^{1 / 2}$

Thus, for $\lambda=10 \mu \mathrm{m}\left(\mathrm{CO}_{2}\right.$ laser $)$ and $I=10^{16} \mathrm{~W} / \mathrm{cm}^{2}$, $v_{0} / c \sim 1$. When the electrons in a plasma are "rattled" this hard, a variety of nonlinear optical effects readily manifest themselves. These include harmonic generation, simulated Brillouin and Raman scattering, degenerate and resonant four wave mixing, self-focusing and parametric amplification. These phenomena are characterized by the generation of one or more scattered e.m. waves that may have a different frequency and direction than the incident e.m. wave. In addition, plasmas demonstrate certain unique nonlinear phenomena such as nonlinear mode conversion, parametric decay instability, two-plasmon decay and relativistic self-focusing. The nonlinear optics of plasmas is therefore both extremely rich and challenging for an experimentalist. It's richness is even greater because many of the nonlinear effects can occur simultaneously in a plasma. Also, when the plasma modes, characteristic of many of these instabilities, become large kinetic effects and wave-wave interactions become extremely important. It is therefore a challenge to isolate and study these effects unambiguously.
On the other hand, the plasma medium can be directly probed using coherent Thomson scattering technique which allows us to "watch" even the most transient interaction; that occurring on $\omega_{p}^{-1}$ timescale. In this paper, experiments carried out by the author and his collaborators in the field on nonlinear optics of plasmas over the past decade are summarized and future directions for this field are outlined.

\section{Beat wave plasma interaction}

Many of the nonlinear-optical effects can be understood in terms of the plasma response to an amplitude modulated or a beat e.m. wave. Consider two e.m. waves $\left(\omega_{0}, \boldsymbol{k}_{0}\right)$ and $\left(\omega_{1}, \boldsymbol{k}_{1}\right)$ propagating through the plasma. The frequency and wave number matching give

$\omega_{0}-\omega_{1}=\omega_{\mathrm{g}} \quad \boldsymbol{k}_{0}-\boldsymbol{k}_{1}=\boldsymbol{k}_{\mathrm{g}}$

where $\omega_{\mathrm{g}}$ and $k_{\mathrm{g}}$ are the frequency and wavenumber of the plasma "grating". Now if $\omega_{\mathrm{g}}=0$ the grating is a standing density wave and the process is known as degenerate excitation. If $\omega_{\mathrm{g}}=\omega_{\mathrm{ac}}=k_{\mathrm{g}} v_{\mathrm{ac}}$, the grating is an ion acoustic wave and the process is known as optical mixing. Here $v_{\mathrm{ac}}$ is the ion acoustic velocity. If $\omega_{\mathrm{g}}=\omega_{\mathrm{p}}$ the grating is now an electron plasma wave (also optical mixing) whereas if $\omega_{\mathrm{g}}=2 \omega_{\mathrm{p}}$ we still have a plasma wave but the process is known as parametric amplification. As in nonlinear optics, the plasma grating can scatter either of the two e.m. waves or a third e.m. wave producing a scattered wave that may have a different frequency and direction than the incident waves. In the nonlinear optics formulation, when the intensities of the incident e.m. waves becomes large, the induced polarization $P(E)$ is expanded as

$P(E)=\chi E+\chi_{2} E^{2}+\chi_{3} E^{3}+\cdots$.

As in a centrosymmetric medium, $\chi_{2}$ in a uniform, unmagnetized and underdense plasma is negligible and harmonic generation can be neglected. The third order susceptibility of the plasma $\chi_{3}$ has its physical origin in the ponderomotive force and the thermal force. The general expression for $\chi_{3}$ is given by Federici and Mansfield [1] as

$\chi_{3}=\frac{n_{0} / n_{\mathrm{c}}\left(\omega_{\mathrm{m}}\right) N_{\mathrm{c}}\left(\omega_{\mathrm{p}}\right)}{8(4 \pi)^{2} T_{\mathrm{e}}\left(1-D_{0}\right)}(1+A)$

where the real portion of the denominator vanishes when $\omega_{\mathrm{g}}=k_{\mathrm{g}} v_{\mathrm{ac}}$ and $A \sim N_{0} v_{\mathrm{c}} / \kappa k_{\mathrm{g}}^{2}$ is the thermal force enhancement factor. All the other symbols are as defined in Ref. [1]. Physically, the first term in the bracket is the contribution to $\chi_{3}$ due to the ponderomotive force $F_{\mathrm{NL}} \sim-\omega_{\mathrm{p}}^{2} / \omega^{2} \nabla\left\langle E^{2} /\right.$ $8 \pi>$ which forces the plasma electrons from regions of high intensity to the regions of low intensity, thus forming the grating. The thermal enhancement of this grating occurs if 
Table I. Laser and plasma conditions for various nonlinear optical experiments

\begin{tabular}{|c|c|c|c|c|c|c|}
\hline & \multicolumn{3}{|l|}{$\mathrm{CO}_{2}$ laser } & \multicolumn{2}{|l|}{ Plasma } & \multirow[b]{2}{*}{ Reference } \\
\hline & Pulselength (ns) & Intensity $\left(\mathrm{W} / \mathrm{cm}^{2}\right)$ & Gas & Density $\left(\mathrm{cm}^{-3}\right)$ & Temperature (eV) & \\
\hline DFWM & 150 & $<10^{8}$ & $\mathrm{Ar}$ & $<10^{17}$ & 4 & 7 \\
\hline RFWM & 150 & $<10^{7}$ & $\mathrm{Ar}, \mathrm{H}$ & $<10^{17}$ & $\sim 4$ & 10 \\
\hline SBS & 50 & $<10^{11}$ & $\mathrm{H}, \mathrm{N}_{2}, \mathrm{He}$ & $2 \times 10^{17}$ & $20-30$ & 12 \\
\hline SRS & 2 & $10^{13}$ & $\mathrm{H}$ & $2-6 \times 10^{16}$ & $20-30$ & 13 \\
\hline $\begin{array}{l}\text { Colinear } \\
\text { optical mixing }\end{array}$ & 2 & $5 \times 10^{13}$ & $\mathrm{H}$ & $10^{17}$ & $20-30$ & 21 \\
\hline Mode coupling & 2 & $5 \times 10^{13}$ & $\mathrm{H}$ & $10^{17}$ & $20-30$ & 23 \\
\hline $\begin{array}{l}\text { Resonent self } \\
\text { focusing }\end{array}$ & 150 & $<10^{9}$ & $\mathrm{Ar}$ & $6 \times 10^{16}$ & 5 & 19 \\
\hline $\begin{array}{l}\text { Parametric } \\
\text { amplification }\end{array}$ & $2 \mathrm{~ns}$ & $10^{13}$ & $\mathrm{H}$ & $2.5 \times 10^{16}$ & $20-30$ & 25 \\
\hline $\begin{array}{l}\text { Beat wave } \\
\text { acceleration }\end{array}$ & $<400 \mathrm{ps}$ & $<10^{14}$ & $\mathrm{H}$ & $6 \times 10^{16}$ & $20 \mathrm{eV}$ & 22 \\
\hline
\end{tabular}

the mean free path of the plasma electrons is smaller than the grating wavelength, because plasma is heated more in the regions of the beat wave where the intensity is high. In order to maintain pressure balance with the nearby region where the beat pattern has an intensity minimum, the plasma density (where the intensity and therefore heating is the largest) drops thus reinforcing the grating.

If the intensity of the incident e.m. wave is large enough, plasma gratings can be excited parametrically [2, 3] from noise while at the same time amplifying the second e.m. wave. Now, if the plasma grating is an ion wave the process is known as Brillouin [4] scattering whereas if it is an electron plasma wave it is known as Raman scattering [5]. If the plasma grating is a standing ion/electron density wave, the process is known as self-focusing [6]. In this paper, we shall discuss the various nonlinear optical effects that occur in a very underdense $\left(n_{0} \ll n_{\mathrm{c}} / 4\right)$ plasma. The laser and the plasma parameters used in our experiments are given in Table I.

\section{Experiments on nonlinear optical effects in plasmas}

\subsection{Degenerate four wave mixing ( $D F W M$ )}

Perhaps the simplest and most readily observed example of a nonlinear optical effect in a plasma is degenerate four wave mixing [7], which is important because of its application to phase conjugation [8]. In DFWM, two counter-propagating pumps and one probe beam of identical frequencies interacts within the nonlinear optical medium, to generate a backward (to the probe) traveling signal wave that is the phase conjugate of the probe beam. The phase matching conditions for DFWM are $\omega_{\mathrm{f}}+\omega_{\mathrm{b}}-\omega_{\mathrm{p}}-\omega_{\mathrm{s}}=0$ and $\boldsymbol{k}_{\mathrm{f}}+\boldsymbol{k}_{\mathrm{b}}-$ $\boldsymbol{k}_{\mathrm{p}}-\boldsymbol{k}_{\mathrm{s}}=0$, where $\mathrm{f}, \mathrm{b}, \mathrm{p}$ and s denote forward, backward, probe and signal waves respectively, and the grating is a non propagating ion density wave.

We have observed DFWM in a $n / n_{\mathrm{c}} \leqslant 10^{-2}$ argon plasma using a single mode $\mathrm{CO}_{2}$ laser. Theory predicts that the signal reflectivity $R$ should scale as

$R \propto \lambda^{10} n_{0}^{6} I_{\mathrm{f}} I_{\mathrm{b}} L^{2} / T_{\mathrm{e}}^{10}$

provided that we are in a regime where the thermal force contribution to the grating formation dominates that due to the ponderomotive force. In our experiments, which were carried out using pump intensities of $3-100 \mathrm{MW} / \mathrm{cm}^{2}$ and probe intensities of $1-10 \mathrm{MW} / \mathrm{cm}^{2}$ and a $150 \mathrm{~ns}$ long laser pulse, we found that $R \propto I_{\mathrm{f}}$ and $I_{\mathrm{b}}$ but nearly independent of $I_{\mathrm{p}}$, as expected. Furthermore, at the highest pump intensities a trend towards saturation was observed and attributed to laser heating of the plasma. The reflectivity was also found to scale as $n_{0}^{6}$, further confirming the importance of collisional effects in allowing the thermal enhancemment of the gratings. We also found that the pump-pump grating, which was much stronger than the two pump-probe gratings did not adversely affect the signal reflectively. Some qualitative features expected from a phase conjugate beam were also observed. Since $\gamma_{3}$ and therefore $R$ scale as $\lambda^{10}$, collisional plasmas appear to be quite promising for phase conjugation via DFWM in the microwave regime.

\subsection{Resonant or nearly-degenerate four wave mixing $(R F W M)$}

The third order susceptibility given by eq. (4) has a resonance when the grating frequency is equal to the ion acoustic frequency. We therefore expect an enhancement of the signal reflectivity in a four wave mixing experiment when the pump and the probe frequencies are chosen such that their frequency difference is the ion acoustic frequency. It can be shown that the density fluctuation level of the grating in such a case is given by

$\frac{n_{1}}{n_{0}} \sim\left(\frac{n_{1}}{n_{0}}\right)_{\text {ponderomotive }}(1+A) \times \frac{k_{\mathrm{g}} v_{\mathrm{ac}}}{\mathrm{i} v_{\mathrm{i}}}$

and

$\left(\frac{n_{1}}{n_{0}}\right)_{\text {ponderomotive }} \sim \frac{v_{0_{\mathrm{f}, \mathrm{p}}} v_{0, \mathrm{p}}}{v_{\mathrm{th}}^{2}}$

where, $v_{\text {th }}$ is electron thermal velocity and $v_{i}$ is the in Landau damping rate. Since the density fluctuation level of the ion grating on the ion acoustic resonance is larger by a factor $(\operatorname{Re} \omega / \operatorname{Im} \omega)$ which in turn is a function of $Z T_{\mathrm{e}} / T_{\mathrm{i}}$, we can in principle get the electron to ion temperature ratio from the enhancement of the signal reflectivity on resonance. Also, since the resonant frequency $k_{\mathrm{g}} v_{\mathrm{ac}}$ is a function of $\sqrt{\left(Z T_{\mathrm{e}}+3 T_{\mathrm{i}} / M\right)}$ we can obtain $T_{\mathrm{e}}$ and $T_{\mathrm{i}}$ separately if the ionization state is known.

We have observed [10] this resonant enhancement of the signal reflectivity by beating the pump and the probe beams in the argon plasma used in DFWM experiments at the ion 
acoustic frequency. The signal enhancement is only a factor of about 3 over the degenerate case suggesting that the plasma ions and electrons are close to thermal equilibrium.

\subsection{Stimulated Brillouin scattering (SBS)}

If the pump laser beam is intense enough, the threshold for simulated Brillouin scattering (SBS) can be exceeded [11] leading to back and side-scattered [10] light from ion waves that grow from noise density fluctuations in the plasma. We have studied the SBS backscatter process in great detail. In our experiments, we measured the exponential growth with incident laser intensity of the SBS backscattered light from non thermal noise and its eventual saturation at a $5-10 \%$ level [11]. In order to understand the saturation mechanism, we have studied the SBS ion wave spectrum, $S(\boldsymbol{k}, \omega)$ directly using ruby Thomson scattering [12]. For our experimental conditions, the SBS instability is in the convective regime and in the strong damping case, $Z T_{\mathrm{e}} / 3 T_{1}=0(1)$, we expect the ion wave amplitude to decrease exponentially with distance $x$ from saturation level to noise level in the direction of the laser beam. Thus

$\left.\frac{n_{\mathrm{i}}(x)}{n_{0}}\right|_{\mathrm{SBS}}=\left(N R^{1 / 2} / \beta L\right) \exp (-N \lambda / 2 L)$

where $\beta=\left(n_{0} / n_{\mathrm{c}}\right)\left(\pi / \lambda_{0}\right)$ and $N$ is the number of e-foldings growth. Our spatially and temporarily resolved Thomson scattered measurements indeed showed an ion wave exponentiating from noise towards the pump beam. We also mapped out the complete $(\omega, k)$ spectrum. It showed discrete harmonics up to the third harmonic. No signs of ion turbulence or half harmonics were evident. Harmonic generation, however, was not found to be the saturation mechanism; the ion wave saturation occurs because of the non-linear frequency shift caused by ion trapping as the ion wave amplitude becomes greater than a few percent.

\subsection{Stimulated Raman scattering (SRS)}

Stimulated Raman scattering (SRS) has a higher threshold than SBS in a plasma basically because plasma waves are more dispersive. However, with higher pump intensities, Raman scattering readily occurs and like SBS, SRS has the maximum gain in the backward direction. The backscattered light is frequency shifted by the Bohm-Gross frequency. In our plasma, the SRS is stronger than or equal in strength compared to SBS for densities less than $5 \times 10^{-3} n_{\mathrm{c}}$. For densities greater than $0.01 n_{\mathrm{c}}$ SBS dominates.

We have used the SRS instability to confirm one of the basic prediction of nonlinear plasma wave theory: that as an electron plasma wave grows, its waveform steepens [13]. Equivalently, the harmonic components of the density perturbation increase in ampitude relative to the fundamental. For the specific case of plasma waves driven by SRS, the harmonic amplitude may be expressed in terms of the fundamental as [14]

$n_{\mathrm{m}} / n_{0}=\alpha_{\mathrm{m}}\left(n_{1} / n_{0}\right)^{m}$

where $\alpha=m^{m} / 2^{(m-1)} m !$. This result is for the cold plasma and the coefficient is modified somewhat in a plasma with a finite temperature [15]. Using Thomson scattering diagnostic, we have mapped the entire spectrum $S(\boldsymbol{k}, \omega)$ of the coherent electron plasma waves and found sharp peaks at $\left(\omega_{\mathrm{p}}, k_{\mathrm{p}}\right)_{n}$ where $n=1,2$ and 3 . Measurements of their relative ampli- tude agreed well with the predictions of nonlinear, warmplasma wave-steepening theory up to the maximum observed amplitude of the fundamental component $n_{1} / n_{0} \sim 16 \%$.

\subsection{Coupling betweem SBS and SRS}

In many experimental situations SBS and SRS may occur simultaneously in space and time. The coupling between these two instabilities can reduce the growth rate of the SRS instability because the ion wave ripples the plasma and modifies the plasma wave dispersion relation, allowing plasma wave spatial harmonics to be generated [16]. These spatial harmonics cannot $\omega$ and $k$ match with the incident e.m. wave (unlike spatiotemporal harmonics) and thus represent an enhanced damping for the SRS instability.

A signature of mode-coupling is an anti-Stokes satellite in the backscattered spectrum with a frequency $\omega_{0}+\omega_{\mathrm{p}}$ as opposed to the usual Stokes satellite characteristic of Raman backscatter at $\omega_{0}-\omega_{p}$. It arises from Thomson scattering of the incident light wave from a counter-propagating coupled mode. We have observed both the anti-Stokes satellite and the backward propagating plasma wave (coupled mode) only when SBS and SRS are simultaneously occurring [17]. We believe that mode coupling is a ubiquitous process and may be the first stage of what finally leads to Langmuir collapse [18].

\subsection{Resonant self-focusing}

When laser light containing two frequencies $\omega_{1}$ and $\omega_{2}$ impinges on a plasma with $\omega_{\mathrm{p}}=\omega_{1}-\omega_{2}$ plasma waves are resonantly excited. This process is often referred to as optical mixing. The ponderomotive force of these waves can be much larger than that of the beam and the self-focusing effect is greatly enhanced [19]. This is because

$F_{\mathrm{NL}}$ (light) $=-\left(\omega_{0}^{2} / \omega_{0}^{2}\right) \nabla\left\langle E_{0}^{2}\right\rangle / 8 \pi$

whereas

$F_{\mathrm{NL}}($ plasmon $)=-\nabla\left\langle E_{\mathrm{p}}^{2}\right\rangle / 8 \pi$.

Here $E_{\mathrm{p}}$ is the plasmon electric field which from Poisson's equation can be shown to be $\left|E_{\mathrm{p}}\right|=4 \pi n_{1} / k_{\mathrm{p}}$. Substituting $v_{0}=e E_{0} / m \omega_{0}$ and $v_{\phi}=\omega_{\mathrm{p}} / k_{\mathrm{p}} \sim c$ we obtain the amplification factor

$A=\frac{F_{\mathrm{NL}}(\text { plasmon })}{F_{\mathrm{NL}}(\text { light }}=\left(\frac{n_{1} / n_{0}}{v_{0} / c}\right)^{2}$.

If we assume that $n_{1} / n_{0}$ for a resonantly excited plasmon is limited by relativistic detuning [20] to

$\left(\frac{n_{1}}{n_{0}}\right)_{\max }=\left(\frac{16}{3} \frac{v_{0}^{2}}{c^{2}}\right)^{1 / 3}$

then, if we assume equal pump strengths the amplification factor

$A=\left(\frac{16}{3} \frac{c}{v_{0}}\right)^{2 / 3}$

which is typically much greater than 1 , for $v_{0} / c \ll 1$. This large ponderomotive force of the excited plasma wave then effectively creates a depression of the plasma density on the beam axis, and the resultant refractive index profile causes the laser beams to self-focus even though the laser intensities are below the ponderomotive or thermal self-focusing threshold. 
With use of $\mathrm{CO}_{2}$ laser light of only $5 \times 10^{9} \mathrm{~W} / \mathrm{cm}^{2}$ intensity and a plasma at $0.6 \%$ of critical density, this resonant self-focusing effect is observed only when the density is close to the resonant value by monitoring the refraction of the laser beam emanating from the focal region.

\subsection{Relativistic plasmon generation and saturation}

In colinear optical mixing, the phase velocity of the plasma wave $v_{\phi}$ is approximately the speed of light $c$. Thus the corresponding Lorentz factor $\gamma_{\phi}=\left(1-v^{2} / c^{2}\right)^{-1 / 2} \cong \omega_{0} / \omega_{p}$. Using Poisson's equation it is easy to show that the maximum longitudinal field of such a wave (approximated by using $\left.n_{1} / n_{0}=1\right)$ turns out to be $\sqrt{n_{\mathrm{e}}\left(\mathrm{cm}^{-3}\right)} \mathrm{V} / \mathrm{cm}$. Thus, for instance, the longitudinal electric field of a plasma wave with a background density of $10^{16} \mathrm{~cm}^{-3} \mathrm{can}$ be as high as $10^{4} \mathrm{MV} / \mathrm{m}$. Thus there is considerable interest in determining if such waves can be used for ultrahigh gradient acceleration of electrons. It can be shown that the energy gain by a trapped electron in such a wave is

$\Delta W=\varepsilon \gamma_{\phi}^{2} m c^{2}$

where $\varepsilon$ is the fractional density modulation of the plasma wave. For instance, $\varepsilon=0.2$ and $\gamma_{\phi}=100$ would give an energy gain $\Delta W=1 \mathrm{GeV}$. If a $1 \mu \mathrm{m}$ laser is used, $\gamma_{\phi}=100$ implies a plasma density of $10^{17} \mathrm{~cm}^{-3}$ and an accelerating gradient of $6.3 \mathrm{GV} / \mathrm{m}$.

In our experiments a relativistic plasma wave was excited [21] by beating the $9.6 \mu \mathrm{m}$ and $10.6 \mu \mathrm{m}$ lines of a $\mathrm{CO}_{2}$ laser, which a modest intensity of $2 \times 10^{13} \mathrm{~W} / \mathrm{cm}^{2}$. The plasma wave electric field was inferred from the Thomson scattering of a probe laser beam to be $10^{3} \mathrm{MeV} / \mathrm{m}$, a substantial improvement over the present benchmark gradient for accelerators. In addition, Stokes and anti-Stokes sidebands in the electromagnetic spectrum were observed, as was a clear density resonance in the amplitude of the Stokes sideband.

A scaled up experiment designed to demonstrate controlled acceleration of externally injected electrons is currently underway at UCLA [22].

A new mechanism, which saturated the beat excited plasma waves, was discovered which can saturate the plasmon amplitude to that below the relativistic saturation value [23]. The relativistic plasma wave saturates, on the time scale of a few picoseconds, by coupling to other plasma modes that have a much lower phase velocity, via an ion ripple due to SBS. These lower phase velocity modes can be Landau damped leading to irreversible loss of energy from the original relativistic plasma wave, which of course has no Landau damping. In our experiments, coupled modes with phase velocities up to $\omega_{\mathrm{p}}+n k_{\mathrm{i}}$ where $n= \pm 1,2,3$ were seen. Here $k_{\mathrm{i}}$ is the wavenumber of the ion ripple and $k_{\mathrm{p}} / k_{\mathrm{f}} \sim 0.05$. As a result of mode coupling, the saturated amplitude of the plasma wave [24] is given by

$E_{\text {sat }}=\frac{1}{\varepsilon} \frac{v_{0}^{2}}{c^{2}} f(p)$

where $\varepsilon$ is the ripple size and $f(p)=p+\left(1+p^{2}\right)(2+$ $\left.p^{2}\right)^{-1 / 2}$ and $p=(3 / \varepsilon)\left(k_{i} \lambda_{\mathrm{D}}\right)^{2}$. Thus, neglecting pump risetimes, saturation by mode coupling dominates over that due to relativistic detuning whenever $v_{0}^{2} / c^{2}<[1.6 \varepsilon / f(p)]^{3 / 2}$ which may be applicable to many experimental situations.

\subsection{Parametric amplifier}

When an oscillator is driven at twice its natural frequency, the amplitude of the oscillator grows exponentially as in a parametric instability rather than secularly as in resonant optical mixing [25]. Using the same experimental set up as that used for the resonant generation of relativistic plasmons, we have observed this parametric amplification process by adjusting the plasma density to one fourth the resonant value such that the drive frequency is twice the plasma frequency. The plasma response was determined by using Thomson scattering. As expected, the Thomson scattered spectrum showed a sharp peak at the drive frequency $\Delta \omega$, an enhanced response at $\omega_{p}$ or $\Delta \omega / 2$, a peak due to scattering from SBS ion wave and a smaller peak due to mode coupling between the ion wave and the parametrically amplified plasma wave at $\omega_{\mathrm{p}}$.

In future work, we propose to measure the exponential growth of the plasma wave and also any hysteresis/bistable behaviour as $\omega_{\mathrm{p}}$ is varied with respect to $\Delta \omega$.

\subsection{Self-focusing and filamentation of laser beams in plasmas}

For modest laser intensities, there are two mechanisms that can lead to whole beam self-focusing or small-scale filamentation: ponderomotive and thermal [26]. In a weakly collisional plasma, ponderomotive beam break up can be understood by considering a beam that is non-uniform in the transverse direction. If such a laser beam is incident on the plasma, then the ponderomotive force exists which pushes the plasma electrons out of the region of highest laser intensity causing locally a density depression in the electron density $n_{0}$. As ions follow the electrons, the refractive index $n$ is raised in the region since $n=\left(1-\omega_{\mathrm{p}}^{2} / \omega_{0}^{2}\right)^{1 / 2}$ and the phase velocity of the light is slowed down causing the light wave to be focused in this region and increasing the irradiance as the beam propagates.

Thermal self-focusing, on the other hand, is caused by localized heating due to collisional absorption of a nonuniform laser beam. Since the thermal pressure $\left(n_{0} k T_{\mathrm{e}}\right)$ of the plasma that is locally heated where the intensity is higher, is greater than its surroundings, it induces faster hydrodynamic expression in order to achieve a pressure balance. The plasma density in the locally heated regions thus decreases leading to an increase in the refractive index. This in turn concentrates the laser in these regions which further exacerbates the heating.

We have carried out a series of experiments in which plasma density perturbations thought to be the result of both ponderomotive and thermal self-focusing were diagnosed using a dark field technique [27]. This technique has subsequently been used at Lawrence Livermore National Laboratory to conclusively demonstrate plasma density striations caused by intentionally superimposed intensity pattern on the beam [28].

In a separate experiment we observed azimuthal periodic break-up of a radially modulated $0.35 \mu \mathrm{m}$ laser beam in plasmas produced from solid targets [29]. The break-up, observed in X-ray pinhole images of the plasma, was more severe in gold plasmas compared to glass or aluminium plasmas and occurred at rather modest laser intensities of $\sim 5 \times 10^{12} \mathrm{~W} / \mathrm{cm}^{2}$. These intensities were above the thermal filamentation threshold, but two orders of magnitude below 
the ponderomotive threshold supporting the notion that thermal filamentation was operative in our experiments.

\subsection{Other nonlinear optical effects in plasmas}

Harmonic generation [30], non-linear mode conversion [31], parametric decay [32], oscillating two-stream instability [33] and two plasmon decay [34] are some of the other non-linear optical effects that are readily observed in plasmas. All except, the two-plasmon decay occur at the critical density where $\omega_{0}=\omega_{\mathrm{p}}$. The latter occurs at the quarter critical density when $\omega_{0}=2 \omega_{\mathrm{p}}$. Since we are concerned with plasmas that are very underdense $\omega_{\mathrm{p}} \ll \omega_{0} / 2$, these effects are not observed in the experiments described in this paper. Nevertheless, in laser fusion experiments, these effects are readily seen and indeed are extremely important either diagnostically or in a more direct way.

\section{Future directions}

The continued development of a variety of laser technologies has opened new opportunities in the field of non-linear optics of plasmas. Lasers with ever increasing output powers and ever shorter pulselengths are becoming commonplace. A glance at Table I shows that our experiments todate have been carried out with laser powers less than $100 \mathrm{GW}$ and laser pulsewidths greater than about $400 \mathrm{ps}$. It is therefore interesting to ask what new physics can be studied with pico and perhaps femtosecond high intensity laser pulses. Such lasers are characterized by the following features:

(a) The laser electric field will exceed the binding field of the atomic electrons.

(b) The pulselengths can be smaller than the ion plasma period and or the collisionless skin depths.

(c) The electromagnetic $v_{0} / c$ may exceed unity.

The first feature implies that, highly uniform, highly ionized, cold plasmas could be produced via multi-photon ionzation [35]. Such plasmas are ideally suited for recombination X-ray laser schemes [36]. The second feature will allow a whole host of relativistic, non-linear phehonema to be studied. These includes relativistic self-focusing [37], magnetic field generation [38], overdense penetration [39], and stochastic heating [38]. The third feature allows experiments in which the ions are essentially immobile to be carried out. An important example of this is the generation of a laser excited "wake" in a plasma [40]. This process is most efficient if the laser pulse is shorter than $\pi c / \omega_{\mathrm{p}}$. In particular, a laser wakefield which is basically a plasma oscillation, excited using a highly relativistic laser pulse is expected to be an excellent collective particle accelerator. Finally, the logical extension of the optical mixing experiments described in this paper would be the non-resonant excitation to study bistable behaviour and chaos in plasmas [41].

\section{Acknowledgements}

The work discussed in this paper is the result of cooperative effort by my many colleagues. In particular, I would like to thank Prof. J. M. Dawson and Prof. F. F. Chen, Drs C. Clayton, T. Katsouleas, W. B. Mori, C. Darrow, Y. Kitagawa, D. Umstadter, H. Figueroa and $\mathrm{Mr}$ R. Williams and $\mathrm{Mr} \mathrm{W}$ Leemans. This work was supported by DOE contract no. DE-AS03-83ER40120, DOE grant no. DE-FG03-87ER13752, ONR contract no. N00014-86-K-0585, UC Nuclear Science CALCOR Funds and LLNL University Research Initative.

\section{References}

1. Federici, J. and Mansfield, D. K., J. Opt. Soc. Am. B3, 1588 (1986).

2. Drake, J. F., Kaw, P. K., Lee, Y. C., Schmidt, G., Liu, C. S. and Rosenbluth, M. N., Phys. Fluids 17, 778 (1974).

3. Drake, J. F. and Lee, Y. C., Phys. Rev. Lett. 31, 1197 (1973).

4. Forslund, D. W., Kindel, J. M. and Lindman, E. L., Phys. Fluids 18, 1002 (1975).

5. Watt, R. G., Brooks, R. D. and Pietryzyk, Z. A., Phys. Rev. Lett. 41, 170 (1978)

6. Kaw, P. K., Schmidt, G. and Wilcox, T., Phys. Fluids 1522 (1973).

7. Kitagawa, Y., Savage, R. L. and Joshi, C., Phys. Rev. Lett. 62, 151 (1989).

8. Optical Phase Conjugation (Edited by R. A. Fisher). Academic, New York (1983).

9. Williams, E. A., Lininger, D. M. and Goldman, M. V., To be published in Phys. Fluids.

10. Kitagawa, Y. and Joshi, C., To be published.

11. Herbst, M. J., Clayton, C. E. and Chen, F. F., Phys. Rev. Lett. 43, 159 I (1979)

12. Clayton, C. E., Joshi, C. and Chen, F. F., Phys. Rev. Lett. 1656 (1983).

13. Umstadter, D., Williams, R. L., Clayton, C. E. and Joshi, C., Phys. Rev. Lett. 59, 292 (1977).

14. Koch, P. and Albritton, J., Phys. Rev. Lett. 34, 1616 (1975).

15. Kuz'menkov, L. S., Solokov, A. A. and Trubachev, O. O., Sov. Phys. J. 26, 1076 (1984).

16. Figueroa, H. and Joshi, C., Phys. Fluids 30, 2294 (1987).

17. Umstadter, D., Mori, W. B. and Joshi, C., Phys. Fluids B1(1), 183 (1989).

18. Rose, H. A., Dubois, D. F. and Bezzerides, B., Phys. Rev, Lett. 58, 2547 (1987).

19. Joshi, C., Clayton, C. E. and Chen, F. F., Phys. Rev. Lett. 48, 874 (1982).

20. Rosenbluth, M. N. and Liu, C. S., Phys. Rev. Lett. 29, 701 (1972).

21. Clayton, C. E., Joshi, C., Darrow, C. and Umstadter, D., Phys. Rev Lett. 54, 2343 (1985)

22. Joshi, C., Proceedings of the Particle Accelerator Conference, Chicago.

23. Darrow, C., Umstadter, D., Katsouleas, T., Mori, W. B., Clayton, C. E. and Joshi, C., Phys. Rev. Lett. 56, 2629 (1986).

24. Darrow, C., Mori, W. B., Katsouleas, T., Joshi, C., Umstadter, D. and Clayton, C. E., IEEE Trans. Plasma Sci. PS-15, 107 (1987).

25. Joshi, C., Proceedings of the Symposium on Advanced Accelerator Concepts, Madison, Wisconsin (1986), AIP Conf. Proc. No 156, Ed. F. Mills (1986).

26. Kruer, W. L., in Comments on Plasma Physics and Controlled Fusion 9, 63 (1985).

27. Joshi, C., Clayton, C. E., Yasuda, A. and Chen, F. F., J. App. Phys. 53, 215 (1982).

28. Young, P. E., Baldis, M. A., Drake, R. P., Campbell, E. M. and Estabrook, K. G., Phys. Rev. Lett. 61, 2336 (1985).

29. Joshi, C., Clayton, C. E., Marsh, K., Sakawa, Y. and Savage, R. L., Opt. Comm. 70, 44 (1989).

30. Carman, R. L., Rhodes, C. K. and Benjamin, R. F., Phys. Rev. A24, 2649 (1981).

31. Carman, R. L., Forslund, D. W. and Kindel, J. M., Phys. Rev. Lett. 46, 29 (1981).

32. Bobin, J. L., Decroisette, M., Meyer, B. and Vitel, Y., Phys. Rev. Lett. 30, 594 (1973).

33. Kruer, W., Laser-Plasma Interaction (1988).

34. Baldis, H. A. and Walsh, C. J., Phys. Rev. Lett 47, 1658 (1981).

35. Dangor, A. E., Dymoke-Bradshaw, A. K. L., Dyson, A., Garvey, T. and Mitchell, L., Rutherford Laboratory Report RAL-87-041 (1987); Perry, M. D., Szoke, A., Landen, O. L. and Campbell, E. M., Phys. Rev. Lett. 60, 1207 (1988)

36. Perry, M. D., Private communication: Corkum, P. and Burnnett, N. H., Submitted to J. Opt. Soc. Am.

37. Mori, W. B., Joshi, C., Dawson, J. M., Forslund, D. W. and Kindel, J. M., Phys. Rev. Lett. 60, 1298 (1988).

38. Forslund, D., Kindel, J. M., Mori, W. B,, Joshi, C. and Dawson, J. M., Phys. Rev. Lett. 54, 558 (1985).

39. Max, C. E. and Perkins, F., Phys, Rev. Lett. 27, 1342 (1971).

40. Tajima, T. and Dawson, J. M., Phys. Rev. Lett. 43, 267 (1979).

41. Leemans, W. P., Joshi, C. and Clayton, C. E., To be published. 